\title{
Research Article \\ Fixed Point Theorems for Nonlinear Contractions in Menger Spaces
}

\author{
Jeong Sheok Ume \\ Department of Mathematics, Changwon National University, Changwon 641-773, Republic of Korea \\ Correspondence should be addressed to Jeong Sheok Ume, jsume@changwon.ac.kr
}

Received 14 May 2011; Accepted 24 June 2011

Academic Editor: H. B. Thompson

Copyright (c) 2011 Jeong Sheok Ume. This is an open access article distributed under the Creative Commons Attribution License, which permits unrestricted use, distribution, and reproduction in any medium, provided the original work is properly cited.

The main purpose of this paper is to introduce a new class of Cirić-type contraction and to present some fixed point theorems for this mapping as well as for Caristi-type contraction. Several examples are given to show that our results are proper extension of many known results.

\section{Introduction}

Probabilistic metric space has been introduced and studied in 1942 by Menger in USA [1], and since then the theory of probabilistic metric spaces has developed in many directions [2]. The idea of Menger was to use distribution functions instead of nonnegative real numbers as values of the metric. The notion of a probabilistic metric space corresponds to the situation when we do not know exactly the distance between two points, we know only probabilistic of metric spaces to be well adapted for the investigation of physiological thresholds and physical quantities particularly in connections with both string and $E$ infinity which were introduced and studied by a well-known scientific hero, El Naschie [3-5].

Ćirić's fixed point theorem [6] and Caristi's fixed point theorem [7] have many applications in nonlinear analysis. These theorems are extended by several authors, see [8$16]$ and the references therein.

In this paper, we introduce a new class of Ćirić-type contraction and present some fixed point theorems for this mapping as well as for Caristi-type contraction. Several examples are given to show that our results are proper extension of many known results.

\section{Preliminaries}

Throughout this paper, we denote by $N$ the set of all positive integers, by $Z^{+}$the set of all nonnegative integers, by $R$ the set of all real numbers, and by $R^{+}$the set of all nonnegative real numbers. We shall recall some definitions and lemmas related to Menger space. 
Definition 2.1. A mapping $F: R \rightarrow R^{+}$is called a distribution if it is nondecreasing left continuous with $\inf \{F(t): t \in R\}=0$ and $\sup \{F(t): t \in R\}=1$. We will denote by $L$ the set of all distribution functions. The specific distribution function $H: R \rightarrow R^{+}$is defined by

$$
H(t)= \begin{cases}0, & t \leq 0 \\ 1, & t>0\end{cases}
$$

Definition 2.2 (see [17]). Probabilistic metric space (PM-space) is an ordered pair $(X, F)$, where $X$ is an abstract set of elements, and $F: X \times X \rightarrow L$ is defined by $(p, q) \rightarrow F_{p, q}$, where $\left\{F_{p, q}: p, q \in X\right\} \subseteq L$, where the functions $F_{p, q}$ satisfied the following:

(a) $F_{p, q}(x)=1$ for all $x>0$ if and only if $p=q$;

(b) $F_{p, q}(0)=0$;

(c) $F_{p, q}=F_{q, p}$;

(d) $F_{p, q}(x)=1$ and $F_{q, r}(y)=1$, then $F_{p, r}(x+y)=1$.

Definition 2.3. A mapping $t:[0,1] \times[0,1] \rightarrow[0,1]$ is called a $t$-norm if

(e) $t(0,0)=0$ and $t(a, 1)=a$ for all $a \in[0,1]$;

(f) $t(a, b)=t(b, a)$ for all $a, b \in[0,1]$;

(g) $t(a, b) \leq t(c, d)$ for all $a, b, c, d \in[0,1]$ with $a \leq c$ and $b \leq d$;

(h) $t(t(a, b), c)=t(a, t(c, d))$ for all $a, b, c \in[0,1]$.

Definition 2.4. Menger space is a triplet $(X, F, t)$, where $(X, F)$ is PM space and $t$ is a $t$ norm such that for all $p, q, r \in X$ and all $x, y \geq 0$,

$$
F_{p, r}(x+y) \geq t\left(F_{p, q}(x), F_{q, r}(y)\right)
$$

Definition 2.5 (see [17]). Let $(X, F, t)$ be a Menger space.

(1) A sequence $\left\{p_{n}\right\}$ in $X$ is said to converge to a point $p$ in $X$ (written as $p_{n} \rightarrow p$ ) if for every $\varepsilon>0$ and $\lambda>0$, there exists a positive integer $M(\varepsilon, \lambda)$ such that $F_{p_{n}, p}(\varepsilon)>1-\lambda$ for all $n \geq M(\varepsilon, \lambda)$.

(2) A sequence $\left\{p_{n}\right\}$ in $X$ is said to be Cauchy if for each $\varepsilon>0$ and $\lambda>0$, there is a positive integer $M(\varepsilon, \lambda)$ such that $F_{p_{n}, p_{m}}(\varepsilon) \geq 1-\lambda$ for all $n, m \in N$ with $n, m \geq$ $M(\varepsilon, \lambda)$.

(3) A Menger space $(X, F, t)$ is said to be complete if every Cauchy sequence in $X$ is converged to a point in $X$.

Definition 2.6 (see [18]). $t$-norm $t$ is said to be of $H$ type if a family of functions $\left\{t^{n}(a)\right\}_{n=1}^{\infty}$ is equicontinuous at $a=1$, that is, for any $\varepsilon \in(0,1)$, there exists $\delta \in(0,1)$, such that $a>1-\delta$ and $n \geq 1$ imply $t^{n}(a)>1-\varepsilon$. The $t$-norm $t=\min$ is a trivial example of $t$-norm of $H$ type, but there are $t$-norms of $H$ type with $t$-norm $\neq$ min (see, e.g., Hadzić [19]). 
Definition 2.7. Let $(X, F, t)$ be a Menger space, and let $T: X \rightarrow X$ be a selfmapping. For each $p \in X, x>0$ and $n \in N$, let

$$
\begin{gathered}
M(p, x, n)=\min \left\{F_{T^{k} p, T^{l} p}(x): k, l \leq n \text { and } k, l \in Z^{+}\right\}, \\
M_{1}(p, x, n)=\min \left\{F_{T^{k} p, T^{l} p}(x): k, l \leq n \text { and } k, l \in N\right\}, \\
M_{2}(p, x, n)=\min \left\{F_{p, T^{l} p}(x): l \leq n \text { and } l \in N\right\}, \\
O(p, n)=\left\{T^{k} p: k \leq n \text { and } k \in Z^{+}\right\}, \\
O(p, \infty)=\left\{T^{k} p: k \in Z^{+}\right\}
\end{gathered}
$$

where it is understood that $T^{0} p=p$.

A Menger space $(X, F, t)$ is said to be $T$ orbitally complete if and only if every Cauchy sequence which is contained in $O(p, \infty)$ for some $p \in X$ converges in $X$.

From Definition 2.1 Definition 2.5, we can prove easily the following lemmas.

Lemma 2.8 (see [20]). Let $(X, d)$ be a metric space, and let $T: X \rightarrow X$ be a selfmapping on $X$. Define $F: X \times X \rightarrow L$ by

$$
[F(p, q)](x) \equiv F_{p, q}(x)=H(x-d(p, q))
$$

for all $p, q \in X$ and $x \in R$, where $\left\{F_{p, q}: p, q \in X\right\} \subseteq$ L. Suppose that $t$-norm $t:[0,1] \times[0,1] \rightarrow$ $[0,1]$ is defined by $t(a, b)=\min \{a, b\}$ for all $a, b \in[0,1]$. Then,

(1) $(X, F, t)$ is a Menger space;

(2) If $(X, d)$ is $T$ orbitally complete, then $(X, F, t)$ is T orbitally complete.

Menger space generated by a metric is called the induced Menger space.

Lemma 2.9. In a Menger space $(X, F, t)$, if $t(x, x) \geq x$ for all $x \in[0,1]$, then $t(a, b)=\min \{a, b\}$ for all $a, b \in[0,1]$.

\section{3. Ćirić-Type Fixed Point Theorems}

In 2010, Cirić proved the following theorem.

Theorem A (see Cirić [9], 2010). Let $(X, F, t)$ be a complete Menger space under a t-norm $t$ of $H$ type. Let $T: X \rightarrow X$ be a generalized $\varphi$-probabilistic contraction, that is,

$$
F_{T p, T q}(\varphi(x)) \geq F_{p, q}(x)
$$

for all $p, q \in X$ and $x>0$, where $\varphi:[0, \infty) \rightarrow[0, \infty)$ satisfies the following conditions: $\varphi(0)=0$, $\varphi(x)<x$, and $\lim _{r \rightarrow x^{+}} \inf \varphi(r)<x$ for each $x>0$. Then, $T$ has a unique fixed point $u \in X$ and $\left\{T^{n}(p)\right\}$ converges to $u$ for each $p \in X$. 
Definition 3.1. Let $(X, F, t)$ be a Menger space with $t(x, x) \geq x$ for all $x \in[0,1]$, and let $T: X \rightarrow$ $X$ be a mapping of $X$. We will say that $T$ is Cirić-type-generalized contraction if

$$
F_{T p, T q}(\varphi(x)) \geq \min \left\{F_{p, q}(x), F_{p, T p}(x), F_{q, T q}(x), F_{p, T q}(x), F_{q, T p}(x)\right\}
$$

for all $p, q \in X$ and $x>0$, where $\varphi:[0, \infty) \rightarrow[0, \infty)$ is a mapping and for all $p, q \in X$ and $x \in R, F_{p, q}(x)$ is the same as in Definition 2.2.

It is clear that $(* 1)$ implies $(* 2)$.

The following example shows that a Cirić-type-generalized contraction need not be a generalized $\varphi$-probabilistic contraction.

Example 3.2. Let $X=[0, \infty), T: X \rightarrow X$ be defined by $T x=x+1$, and let $\varphi:[0, \infty) \rightarrow[0, \infty)$ be defined by

$$
\varphi(x)= \begin{cases}\frac{x}{1+x}, & 0 \leq x \leq 1 \\ x-1, & 1<x\end{cases}
$$

For each $p, q \in X$, let $F_{p, q}: R \rightarrow R^{+}$be defined by $F_{p, q}(x)=H(x-d(p, q))$ for all $x \in R$, where $H$ is the same as in Definition 2.1, and $d$ is a usual metric on $R \times R$. Then, since $\max \{\mid p-q-$ $1|| q-p-1 \mid,\}=|p-q|+1$ for all $p, q \in X$, we have $F_{T p, T q}(\varphi(x)) \geq \min \left\{F_{p, T q}(x), F_{q, T p}(x)\right\}$ for all $p, q \in X$ and $x>0$. Thus,

$$
F_{T p, T q}(\varphi(x)) \geq \min \left\{F_{p, q}(x), F_{p, T p}(x), F_{q, T q}(x), F_{p, T q}(x), F_{q, T p}(x)\right\}
$$

for all $p, q \in X$ and $x>0$, which satisfies $(* 2)$. If $x=2, p=0$ and $q=3 / 2$, then $F_{T 0, T 3 / 2}(\varphi(2))=$ 0 and $F_{0,3 / 2}(2)=1$. Thus, $F_{T 0, T 3 / 2}(\varphi(2))<F_{0,3 / 2}(2)$, which does not satisfy $(* 1)$.

In the next example, we shall show that there exists $T$ that does not satisfy $(* 2)$ with $\varphi(t)=k t, 0<k<1$.

Example 3.3. Let $X=[0, \infty), T: X \rightarrow X$ be defined by $T x=2 x$ and let $\varphi:[0, \infty) \rightarrow[0, \infty)$ be defined by $\varphi(x)=k x, 0<k<1$. For each $p, q \in X$, let $F_{p, q}: R \rightarrow R^{+}$be defined by $F_{p, q}(x)=H(x-d(p, q))$ for all $x \in R$, where $H$ is the same as in Definition 2.1, and $d$ is a usual metric on $R \times R$. If $p=0, q=1$ and $x=2 / k>0$, then for simple calculations, $F_{T 0, T 1}(\varphi(2 / k))=0$ and

$$
\min \left\{F_{0,1}\left(\frac{2}{k}\right), F_{0, T 0}\left(\frac{2}{k}\right), F_{1, T 1}\left(\frac{2}{k}\right), F_{0, T 1}\left(\frac{2}{k}\right), F_{1, T 0}\left(\frac{2}{k}\right)\right\}=1
$$

Therefore, for $p=0, q=1$, and $x=2 / k>0$, the mapping $T$ does not satisfy $(* 2)$. Thus, we showed that there exists $T$ that does not satisfy $(* 2)$ with $\varphi(t)=k t, 0<k<1$. 
Definition 3.4. Let $(X, F, t)$ be a Menger space with $t(x, x) \geq x$ for all $x \in[0,1]$ and let $T: X \rightarrow$ $X$ be a self mapping of $X$. We will say that $T$ is a mapping of type $\mathbb{U}$ if there exists $p \in X$ such that

$$
F_{p, T p}((I-\varphi)(x)) \leq \inf \left\{F_{T^{k} p, T^{l} p}(x): k, l \in Z^{+}\right\} \quad \forall x>0,
$$

where $\varphi:[0, \infty) \rightarrow[0, \infty)$ is a mapping, and $I:[0, \infty) \rightarrow[0, \infty)$ is identity mapping. and $(* 3)$.

The following example shows that $T$ has no fixed point, even though $T$ satisfies $(* 2)$

Example 3.5. Let $X=[0, \infty), T: X \rightarrow X$ be defined by $T x=x+4$, and let $\varphi:[0, \infty) \rightarrow[0, \infty)$ be defined by

$$
\varphi(x)= \begin{cases}\frac{x}{2}, & 0 \leq x \leq 4 \\ x-2, & 4<x .\end{cases}
$$

For each $p, q \in X$, let $F_{p, q}: R \rightarrow R^{+}$be defined by $F_{p, q}(x)=H(x-d(p, q))$ for all $x \in R$, where $H$ is the same as in Definition 2.1, and $d$ is a usual metric on $R \times R$. Then, since $\max \{\mid p-q-$ $4|| q-p-4 \mid,\}=|p-q|+4$ for all $p, q \in X$, we have $F_{T p, T q}(\varphi(x)) \geq \min \left\{F_{p, T q}(x), F_{q, T p}(x)\right\}$ for all $p, q \in X$ and $x>0$. Thus,

$$
F_{T p, T q}(\varphi(x)) \geq \min \left\{F_{p, q}(x), F_{p, T p}(x), F_{q, T q}(x), F_{p, T q}(x), F_{q, T p}(x)\right\}
$$

for all $p, q \in X$ and $x>0$, which implies $(* 2)$. It is easy to see that there exists $p=1 \in X$ such that

$$
F_{p, T p}((I-\varphi)(x)) \leq \inf \left\{F_{T^{k} p, T^{l} p}(x): k, l \in Z^{+}\right\} \quad \forall x>0,
$$

which implies $(* 3)$. But $T$ has no fixed point.

Remark 3.6. It follows from Example 3.5 that $T$ must satisfy $(* 2),(* 3)$, and other conditions in order to have fixed point of $T$.

The following is Ćirić-type fixed point theorem which is generalization of Ćirić's fixed point theorems $[6,9]$.

Theorem 3.7. Let $(X, F, t)$ be a Menger space with continuous $t$ norm and $t(x, x) \geq x$ for all $x \in$ $[0,1]$, let $T$ be a self-mapping on $X$ satisfying $(* 2)$ and $(* 3)$. Let $(X, F, t)$ be $T$ orbitally complete. Suppose that $\varphi: R^{+} \rightarrow R^{+}$is a mapping such that

(i) $\varphi(x)<x$ for all $x>0$ and $\lim _{x \rightarrow \infty}(I-\varphi)(x)=\infty$, where $I: R^{+} \rightarrow R^{+}$is identity mapping,

(ii) $\varphi$ and $I-\varphi$ are strictly increasing and onto mappings,

(iii) $\lim _{n \rightarrow \infty} \varphi^{-n}(x)=\infty$ for each $x>0$, where $\varphi^{-n}$ is $n$-time repeated composition of $\varphi^{-1}$ with itself. 
Then,

(a) $M(p, x, n)=\min \left\{M_{1}(p, x, n), M_{2}(p, x, n)\right\}$ for all $p \in X, x>0$, and $n \in N$,

(b) $M(p, x, n)=M_{2}(p, x, n)$ for all $p \in X, x>0$ and $n \in N$,

(c) $\left\{T^{n} p\right\}$ is Cauchy sequence for each $p \in U$, where

$$
U=\left\{p \in X \mid F_{p, T p}((I-\varphi)(x)) \leq \inf \left[F_{T^{k} p, T^{l} p}(x): k, l \in Z^{+}\right] \forall x>0\right\}
$$

(d) Thas a unique fixed point in X.

Proof. Let $p \in X, x>0$ and $n \in N$ be arbitrary. By Definition 2.2 and Definition 2.7, clearly, we have $M(p, x, n)=\min \left\{M_{1}(p, x, n), M_{2}(p, x, n)\right\}$ which implies (a). From (i), (ii), and (*2), we have

$$
\begin{aligned}
M_{1}(p, \varphi(x), n) & =\min \left\{F_{T^{k} p, T^{l} p}(\varphi(x)) \mid k, l \leq n \text { and } k, l \in N\right\} \\
& =\min \left\{F_{T T^{k-1} p, T T^{l-1} p}(\varphi(x)) \mid k, l \leq n \text { and } k, l \in N\right\} \\
& \geq \min \left\{\operatorname { m i n } \left[F_{T^{k-1} p, T^{l-1} p}(x), F_{T^{k-1} p, T^{k} p}(x), F_{T^{l-1} p, T^{l} p}(x),\right.\right. \\
& \left.\left.F_{T^{k-1} p, T^{l} p}(x), F_{T^{l-1} p, T^{k} p}(x)\right]: k, l \leq n \text { and } k, l \in N\right\} \\
& \geq M(p, x, n) .
\end{aligned}
$$

By virtue of (i), (ii), (3.8), and (a), we obtain

$$
\begin{aligned}
M_{1}(p, x, n) & \geq \min \left\{M_{1}\left(p, \varphi^{-1}(x), n\right), M_{2}\left(p, \varphi^{-1}(x), n\right)\right\} \\
& \geq \min \left\{M_{1}\left(p, \varphi^{-1}(x), n\right), M_{2}(p, x, n)\right\} .
\end{aligned}
$$

By repeating application of (3.9), we have

$$
M_{1}(p, x, n) \geq \min \left\{M_{1}\left(p, \varphi^{-m}(x), n\right), M_{2}(p, x, n)\right\}
$$

Since $M_{1}\left(p, \varphi^{-m}(x), n\right)$ converges to 1 when $m$ tends to infinity, it follows that

$$
M_{1}(p, x, n) \geq M_{2}(p, x, n)
$$

On account of (a) and (3.11), we have $M(p, x, n)=M_{2}(p, x, n)$ for $p \in X, x>0$ and $n \in N$, which implies (b). To prove (c), let $n$ and $m$ be two positive integers with $n<m, p \in U$, and let $x$ be any positive real number. By $(* 2)$ and $(b)$, we have

$$
\begin{gathered}
F_{T^{n} p, T^{m} p}(\varphi(x))=F_{T T^{n-1} p, T T^{m-1} p}(\varphi(x)) \\
\geq \min \left\{F_{T^{n-1} p, T^{m-1} p}(x), F_{T^{n-1} p, T^{n} p}(x), F_{T^{m-1} p, T^{m} p}(x),\right. \\
\left.F_{T^{n-1} p, T^{m} p}(x), F_{T^{n} p, T^{m-1} p}(x)\right\}
\end{gathered}
$$


Abstract and Applied Analysis

$$
\begin{aligned}
& \geq \min \left\{F_{T^{i} T^{n-1} p, T^{i} T^{n-1} p}(x): i, j \leq m-n+1, i, j \in Z^{+}\right\} \\
& =\min \left\{F_{T^{n-1} p, T^{l} T^{n-1} p}(x): l \leq m-n+1, l \in N\right\}
\end{aligned}
$$

$$
\min \left\{F_{T^{n-1} p, T^{l} T^{n-1} p}(\varphi(x)): l \leq m-n+1, l \in N\right\}
$$$$
=\min \left\{F_{T T^{n-2} p, T T^{l+n-2} p}(\varphi(x)): l \leq m-n+1, l \in N\right\}
$$$$
\geq \min \left\{\operatorname { m i n } \left[F_{T^{n-2} p, T^{l+n-2} p}(x), F_{T^{n-2} p, T^{n-1} p}(x), F_{T^{l+n-2} p, T^{l+n-1} p}(x),\right.\right.
$$

$$
\left.\left.F_{T^{n-2} p, T^{l+n-1} p}(x), F_{T^{n-1} p, T^{l+n-2} p}(x)\right]: l \leq m-n+1, l \in N\right\}
$$$$
\geq \min \left\{\min \left[F_{T^{i} T^{n-2} p, T^{j} T^{n-2} p}(x): 0 \leq i, j \leq l+1, i, j \in Z^{+}\right]\right.
$$

$$
\begin{aligned}
& : l \leq m-n+1, l \in N\} \\
\geq \min \left\{F_{T^{i} T^{n-2} p, T^{j} T^{n-2} p}(x): 0 \leq i, j \leq m-n+2, i, j \in Z^{+}\right\} & \min \left\{F_{T^{n-2} p, T^{l} T^{n-2} p}(x): l \leq m-n+2, l \in N\right\} .
\end{aligned}
$$

In terms of (i), (ii), and (3.13), we get

$$
\begin{aligned}
F_{T^{n} p, T^{m} p}(x) & \geq \min \left\{F_{T^{n-1} p, T^{l} T^{n-1} p}\left(\varphi^{-1}(x)\right): l \leq m-n+1, l \in N\right\} \\
& \geq \min \left\{F_{T^{n-2} p, T^{l} T^{n-2} p}\left(\varphi^{-2}(x)\right): l \leq m-n+2, l \in N\right\} .
\end{aligned}
$$

By repeating the same method as in (3.13) and (3.14), we have

$$
\begin{aligned}
F_{T^{n} p, T^{m} p}(x) & \geq \min \left\{F_{p, T^{l} p}\left(\varphi^{-n}(x)\right): l \leq m, l \in N\right\} \\
& \geq \inf \left\{F_{p, T^{l} p}\left(\varphi^{-n}(x)\right): l \in N\right\} .
\end{aligned}
$$

On account of (iii), (*3), (b), (3.15), and Definition 2.2, we have

$$
\begin{aligned}
\lim _{n \rightarrow \infty} \inf \left\{F_{p, T^{l} p}\left(\varphi^{-n}(x)\right): l \in N\right\} & \geq \lim _{n \rightarrow \infty} \inf \left\{F_{T^{k} p, T^{l} p}\left(\varphi^{-n}(x)\right): k, l \in Z^{+}\right\} \\
& \geq \lim _{n \rightarrow \infty} F_{p, T p}\left((1-\varphi)\left(\varphi^{-n}(x)\right)\right)=1 \text { for } x>0 .
\end{aligned}
$$


It follows from (3.15) and (3.16) that

$$
\lim _{n \rightarrow \infty} F_{T^{n} p, T^{m} p}(x)=1 \quad \text { for } p \in U, x>0 .
$$

This implies that $\left\{T^{n} p\right\}$ is a Cauchy sequence for $p \in U$. This is the proof of (c). Since $X$ is $T$ orbitally complete, and $\left\{T^{n} p\right\}$ is a Cauchy sequence for $p \in U,\left\{T^{n} p\right\}$ has a limit $u$ in $X$. To prove (d), let us consider the following inequality;

$$
F_{T u, T^{n+1} p}(\varphi(x)) \geq \min \left\{F_{u, T^{n} p}(x), F_{u, T u}(x), F_{T^{n} p, T^{n+1} p}(x), F_{u, T^{n+1} p}(x), F_{T^{n} p, T u}(x)\right\} \quad \forall x>0 .
$$

Since $\lim _{n \rightarrow \infty} T^{n} p=u$, from (3.18), we get

$$
F_{T u, u}(\varphi(x)) \geq F_{T u, u}(x) \quad \forall x>0 .
$$

In terms of (3.19), (i), (ii), (iii), and Definition 2.2, we deduce that $T u=u$, that is, $u$ is a fixed point of $T$. To prove uniqueness of a fixed point of $T$, let $w$ be another fixed point of $T$. Then $T w=w$. Putting $p=u$ and $q=w$ in $(* 2)$, we get

$$
\begin{aligned}
F_{T u, T w}(\varphi(x)) & =F_{u, w}(\varphi(x)) \\
& \geq \min \left\{F_{u, w}(x), F_{u, T u}(x), F_{w, T w}(x), F_{u, T w}(x), F_{w, T u}(x)\right\} \\
& =F_{u, w}(x) \quad \forall x>0,
\end{aligned}
$$

which gives $u=w$. Thus, $u$ is a unique fixed point of $T$, which implies (d).

Corollary 3.8 (see [6]). let $T$ be a quasicontraction on a metric space $(X, d)$, that is, there exists $k \in(0,1)$ such that

$$
d(T p, T q) \leq k \cdot \max \{d(p, q), d(p, T p), d(q, T q), d(p, T q), d(q, T p)\}, \quad \forall p, q \in X
$$

Suppose that $X$ is $T$ orbitally complete. Then, $T$ has a unique fixed point in $X$.

Proof. Define $F: X \times X \rightarrow L$ by $F(p, q)=F_{p, q}$ for all $p, q \in X$ and $F_{p, q}(x)=H(x-d(p, q))$ for all $p, q \in X$ and $x \in R$, where $H$ and $L$ are the same as in Definition 2.1. Let $t:[0,1] \times[0,1] \rightarrow$ $[0,1]$ be defined by $t(a, b)=\min \{a, b\}$ for all $a, b \in[0,1]$. Let $\varphi:[0, \infty) \rightarrow[0, \infty)$ be defined by

$$
\varphi(x)=k x, \quad 0<k<1 .
$$

Then from Lemma 2.8, $(X, F, t)$ is a $T$ orbitally complete Menger space. It follows from (3.21), Lemma 2.8, Lemma 2.9, and [6, lemma 2] that all conditions of Theorem 3.7 are satisfied. Therefore, $T$ has a unique fixed point in $X$. 
Now we shall present an example to show that all conditions of Theorem 3.7 are satisfied but condition (3.21) in Corollary 3.8 and condition $(* 1)$ in Theorem A are not satisfied.

Example 3.9. Let $X=[-1,1]$ be the closed interval with the usual metric and $T: X \rightarrow X$ and $\varphi: R^{+} \rightarrow R^{+}$be mappings defined as follows:

$$
\begin{gathered}
T p= \begin{cases}0, & -1 \leq p<0, \\
\frac{p}{1+p^{2}}, & 0 \leq p<\frac{4}{5} \quad \text { or } \quad \frac{7}{8}<p \leq 1, \\
-\frac{1}{16} p, & \frac{4}{5} \leq p \leq \frac{7}{8},\end{cases} \\
\varphi(t)= \begin{cases}t-\frac{t^{2}}{8}, & 0 \leq t \leq 1, \\
\frac{7}{8} t, & 1<t .\end{cases}
\end{gathered}
$$

Define $F_{p, q}: R \rightarrow R^{+}$by

$$
F_{p, q}(x)=H(x-|p-q|)
$$

for all $p, q \in X$ and $x \in R$, where $F_{p, q}$ and $H$ are the same as in Definition 2.1 and Definition 2.2. Let $t:[0,1] \times[0,1] \rightarrow[0,1]$ be defined by $t(a, b)=\min \{a, b\}$ for all $a, b \in[0,1]$. Then from Lemma 2.8, $(X, F, t)$ is a $T$ orbitally complete Menger space, and $\varphi$ is continuous function on $R^{+}$which satisfy (i), (ii), and (iii). Clearly $p=0 \in X$ satisfies $(* 3)$. To show that condition $(* 2)$ is satisfied, we need to consider several possible cases.

Case 1. Let $p, q \in[-1,0)$. Then

$$
d(T p, T q)=|T p-T q|=0 \leq \varphi(d(p, q))
$$

Case 2. Let $p \in[-1,0)$ and $q \in[0,4 / 5) \cup(7 / 8,1]$. Then

$$
\begin{aligned}
d(T p, T q) & =|T p-T q|=|T q|=\frac{q}{1+q} \leq q-\frac{q^{2}}{8} \\
& =\varphi(q)=\varphi(|q-T p|)=\varphi(d(q, T p))
\end{aligned}
$$

Case 3. Let $p \in[-1,0)$ and $q \in[4 / 5,7 / 8]$. Then

$$
\begin{aligned}
d(T p, T q) & =|T p-T q|=|T q|=\frac{1}{16} q \leq q-\frac{q^{2}}{8}=\varphi(q) \\
& =\varphi(|q-T p|)=\varphi(d(q, T p)) .
\end{aligned}
$$


Case 4. Let $p, q \in[0,4 / 5) \cup(7 / 8,1]$. Then, by simple calculation,

$$
\begin{aligned}
d(T p, T q) & =|T p-T q|=\left|\frac{p}{1+p}-\frac{q}{1+q}\right| \\
& \leq|p-q|-\frac{|p-q|^{2}}{8}=\varphi(|p-q|) \\
& =\varphi(d(p, q)) .
\end{aligned}
$$

Case 5. Let $p \in[0,4 / 5) \cup(7 / 8,1]$ and $q \in[4 / 5,7 / 8]$. Then

$$
\begin{aligned}
d(T p, T q) & =|T p-T q|=\left|\frac{p}{1+p}-\left(-\frac{1}{16} q\right)\right|=\frac{p}{1+p}+\frac{1}{16} q \\
& \leq \frac{1}{2}+\frac{1}{16} \times \frac{7}{8}=\frac{71}{128} \\
& =\varphi(d(p, q)), \\
\varphi(d(q, T q)) & =\varphi(|q-T q|)=\varphi\left(\left|q-\left(-\frac{1}{16} q\right)\right|\right)=\varphi\left(\frac{17}{16} q\right) \\
& \geq \varphi\left(\frac{4}{5} \times \frac{17}{16}\right)=\frac{17}{20} \times \frac{143}{160}>\frac{71}{128} .
\end{aligned}
$$

Thus,

$$
d(T p, T q) \leq \frac{71}{128}<\frac{17}{20} \times \frac{143}{160}<\varphi(d(q, T q)) .
$$

Case 6. Let $p, q \in[4 / 5,7 / 8]$. Then,

$$
d(T p, T q)=|T p-T q|=\left|\left(-\frac{1}{16} p\right)-\left(-\frac{1}{16} q\right)\right|=\frac{1}{16}|p-q| \leq \varphi(|p-q|)=\varphi(d(p, q)) .
$$

Hence, we obtain

$$
d(T p, T q) \leq \varphi(M(p, q)) \quad \forall p, q \in[-1,1]
$$

where

$$
M(p, q)=\max \{d(p, q), d(p, T p), d(q, T q), d(p, T q), d(q, T p)\}
$$


From (3.25) and (3.34), we have

$$
F_{T p, T q}(\varphi(x)) \geq \min \left\{F_{p, q}(x), F_{p, T p}(x), F_{q, T q}(x), F_{p, T q}(x), F_{q, T p}(x)\right\}
$$

for all $p, q \in X$ and $x>0$, which implies $(* 2)$. Therefore, all hypotheses of Example 3.9 satisfy that of Theorem 3.7. Hence, $T$ has a unique fixed point 0 in $X$. On the other hand, let $k \in(0,1)$ be any fixed number. Then, for $p=0 \in X$ and $q \in X$ with $0<q<\min \{4 / 5,(1 / \mathrm{k})-1\}$, we have

$$
\begin{aligned}
k \cdot \max & \{d(p, q), d(p, T p), d(q, T q), d(p, T q), d(q, T p)\} \\
= & k \cdot d(p, q)<\frac{1}{1+q} d(p, q)=\frac{q}{1+q}=d(0, T q)=d(T p, T q) .
\end{aligned}
$$

Thus,

$$
d(T p, T q)>k \cdot \max \{d(p, q), d(p, T p), d(q, T q), d(p, T q), d(q, T p)\}
$$

which shows that $T$ does not satisfy (3.21).

Finally, in above Example 3.9, we shall show that $T$ does not satisfy $(* 1)$. In fact, we need to show that there are $p, q \in X$ and $x>0$ such that $F_{T p, T q}(\varphi(x))<F_{p, q}(x)$. Let $p=4 / 5$, $q=(4 / 5)-(1 / 100)$, and

$$
x=4-\sqrt{16-8 \times\left(\frac{1}{20}+\frac{79}{179}\right)} .
$$

Then, $1 / 100<x<1, \varphi(x)-|T p-T q|=0$, and $x-|p-q|>0$. Hence, $H(\varphi(x)-|T p-T q|)=0$ and $H(x-|p-q|)=1$. Thus, $F_{T p, T q}(\varphi(x))<F_{p, q}(x)$. Therefore, Theorem 3.7 is a proper extension of Theorem A and Corollary 3.8 .

\section{Caristi-Type Fixed Point Theorems}

The following Lemma plays an important role to prove Caristi-type fixed point theorem which is generalization of Caristi's fixed point theorem [7].

Lemma 4.1. Let $(X, F, t)$ be a Menger space with continuous $t$ norm and $t(x, x) \geq x$ for all $x \in$ $[0,1]$, and let $H: R \rightarrow R^{+}$be the same as in Definition 2.1. Suppose that $g: X \times X \rightarrow R^{+}$and $f: X \rightarrow(-\infty, \infty]$ are mappings satisfying the following conditions:

(1) $g(u, w) \leq g(u, v)+g(v, w)$ for all $u, v, w \in X$,

(2) $f$ is a proper function which is bounded from below,

(3) for any sequence $\left\{u_{n}\right\}_{n=1}^{\infty}$ in $X$ satisfying

$$
\lim _{n \rightarrow \infty}\left\{\sup \left[g\left(u_{n}, u_{m}\right): m>n\right]\right\}=0,
$$


there exists $u_{0} \in X$ such that

$$
\begin{gathered}
\lim _{n \rightarrow \infty} u_{n}=u_{0}, \\
g\left(u_{n}, u_{0}\right) \leq \liminf _{m \rightarrow \infty} g\left(u_{n}, u_{m}\right), \\
f\left(u_{0}\right) \leq \liminf _{n \rightarrow \infty} f\left(u_{n}\right),
\end{gathered}
$$

(4) for any $u \in X$ with $\inf _{v \in X} f(v)<f(u)$, there exists $w \in X-\{u\}$ such that

$$
\begin{gathered}
g(u, w) \leq f(u)-f(w), \\
F_{u, w}(k x) \geq H\left(x-\frac{1}{k}[f(u)-f(w)]\right), \quad \forall x>0 \text { and some } k \in(0,1),
\end{gathered}
$$

where $L$ is the set of all distribution functions,

$$
\left\{F_{p, q}: p, q \in X\right\} \subseteq L, \quad F: X \times X \rightarrow L
$$

is defined by $F(p, q)=F_{p, q}$ for all $p, q \in X$. Then, there exists $w_{0} \in X$ such that $\inf _{v \in X} f(v)=f\left(w_{0}\right)$. Proof. Suppose that

$$
\inf _{v \in X} f(v)<f(u) \quad \forall u \in X
$$

For each $u \in X$, let

$$
S(u)=\{w \in X \mid g(u, w) \leq f(u)-f(w)\} .
$$

Then, by (4), (4.6), and (4.7) $S(u)$ is nonempty for each $u \in X$. From (1) and (4.7), we obtain

$$
S(w) \subseteq S(u), \quad \text { for each } w \in S(u)
$$

For each $u \in X$, let

$$
c(u)=\inf \{f(w) \mid w \in S(u)\} .
$$

Choose $u \in X$ with $f(u)<\infty$. Then from (4.8) and (4.9), there exists a sequence $\left\{u_{n}\right\}_{n=1}^{\infty}$ in $X$ such that for all $n \in N$

$$
u_{1}=u, \quad u_{n+1} \in S\left(u_{n}\right), \quad S\left(u_{n}\right) \subseteq S(u), \quad f\left(u_{n+1}\right)<c\left(u_{n}\right)+\frac{1}{n} .
$$


In virtue of (4.7), (4.9), and (4.10), we have

$$
\begin{aligned}
& g\left(u_{n}, u_{n+1}\right) \leq f\left(u_{n}\right)-f\left(u_{n+1}\right), \\
& f\left(u_{n+1}\right)-\frac{1}{n}<c\left(u_{n}\right) \leq f\left(u_{n+1}\right)
\end{aligned}
$$

for all $n \in N$. In view of (4.11), $\left\{f\left(u_{n}\right)\right\}_{n=1}^{\infty}$ is a nonincreasing sequence of real numbers, and so it converges to some $\beta \in R$. Therefore, due to (4.12),

$$
\beta=\lim _{n \rightarrow \infty} c\left(u_{n}\right)=\lim _{n \rightarrow \infty} f\left(u_{n}\right)
$$

Combining (1) and (4.11), we get

$$
g\left(u_{n}, u_{m}\right) \leq f\left(u_{n}\right)-f\left(u_{m}\right) \quad \forall n, m \in N \text { with } n<m .
$$

On account of (4.13) and (4.14), we have

$$
\lim _{n \rightarrow \infty}\left\{\sup \left[g\left(u_{n}, u_{m}\right): m>n\right]\right\}=0
$$

Thus, by virtue of (3), (4.13), (4.14), and (4.15), there exists $u_{0} \in X$ such that

$$
\begin{gathered}
\lim _{n \rightarrow \infty} u_{n}=u_{0}, \\
f\left(u_{0}\right) \leq \lim _{n \rightarrow \infty} f\left(u_{n}\right)=\beta, \\
g\left(u_{n}, u_{0}\right) \leq \liminf _{m \rightarrow \infty} g\left(u_{n}, u_{m}\right) .
\end{gathered}
$$

Using (4.14), (4.17), and (4.18), we obtain

$$
\begin{aligned}
f\left(u_{0}\right) \leq \beta & =\limsup _{m \rightarrow \infty} f\left(u_{m}\right) \\
& \leq \limsup _{m \rightarrow \infty}\left\{f\left(u_{n}\right)-g\left(u_{n}, u_{m}\right)\right\} \\
& =f\left(u_{n}\right)+\limsup _{m \rightarrow \infty}\left\{-g\left(u_{n}, u_{m}\right)\right\} \\
& =f\left(u_{n}\right)-\liminf _{m \rightarrow \infty} g\left(u_{n}, u_{m}\right) \\
& \leq f\left(u_{n}\right)-g\left(u_{n}, u_{0}\right) .
\end{aligned}
$$

Combining (4.7), (4.9), and (4.19), it follows that $u_{0} \in S\left(u_{n}\right)$ and, hence,

$$
c\left(u_{n}\right) \leq f\left(u_{0}\right), \quad \forall n \in N
$$


Taking the limit in inequality (4.20) when $n$ tends to infinity, we have

$$
\lim _{n \rightarrow \infty} c\left(u_{n}\right) \leq f\left(u_{0}\right) .
$$

In terms of (4.13), (4.17), and (4.21), we deduce that

$$
\beta=f\left(u_{0}\right) .
$$

On the other hand, from (4), (4.6), (4.7), and (4.16), we have the following property:

$$
\text { there exists } w_{1} \in X-\left\{u_{0}\right\} \text { satisfying } w_{1} \in S\left(u_{0}\right) \text {. }
$$

In terms of (4.7), (4.8), (4.9), (4.20), and (4.23), we deduce that

$$
\begin{gathered}
w_{1} \in S\left(u_{n}\right), \quad \forall n \in N, \\
c\left(u_{n}\right) \leq f\left(w_{1}\right) .
\end{gathered}
$$

In view of (4.7), (4.13), (4.22), (4.23), and (4.25), we have

$$
\beta=f\left(w_{1}\right)
$$

Due to (4), (4.22), (4.23), and (4.26), we have the following:

$$
\begin{aligned}
F u_{0}, w_{1}(k x) & \geq H\left(x-\frac{1}{k}\left[f\left(u_{0}\right)-f\left(w_{1}\right)\right]\right) \\
& =H(x) \geq F u_{0}, w_{1}(x) \quad \forall x>0 .
\end{aligned}
$$

By virtue of (4.27), we obtain

$$
F u_{0}, w_{1}(x) \geq F u_{0}, w_{1}\left(k^{-1} x\right), \quad \forall x>0 .
$$

By repeating the application of inequality (4.28), we get

$$
F u_{0}, w_{1}(x) \geq F u_{0}, w_{1}\left(k^{-m} x\right), \quad \forall x>0, m \in N
$$

In terms of (4.29), we deduce that $F u_{0}, w_{1}\left(k^{-m} x\right)$ converges to 1 as $m \rightarrow \infty$ and, hence,

$$
F u_{0}, w_{1}(x)=1, \quad \forall x>0 .
$$


From (4.30) and Definition 2.2, we have $u_{0}=w_{1}$. This is a contradiction from (4.23). Therefore, there exists $w_{0} \in X$ such that

$$
\inf _{v \in X} f(v)=f\left(w_{0}\right)
$$

Theorem 4.2. Let $(X, d)$ be a metric space and let $H, g, L$, and $f$ satisfy conditions (1), (2), and (3) in Lemma 4.1. Suppose that for any $u \in X$ with $\inf _{v \in X} f(v)<f(u)$, there exists $w \in X-\{u\}$ such that

$$
\begin{gathered}
g(u, w) \leq f(u)-f(w), \\
H(k x-d(u, w)) \geq H\left(x-\frac{1}{k}[f(u)-f(w)]\right)
\end{gathered}
$$

for all $x>0$ and some $k \in(0,1)$. Thus, there exists $w_{0} \in X$ such that

$$
\inf _{v \in X} f(v)=f\left(w_{0}\right)
$$

Proof. The proof follows from Lemma 4.1 by considering the induced Menger space $(X, F, t)$, where $t(a, b)=\min \{a, b\}$ and

$$
F_{p, q}(x)=H(x-d(p, q)), \quad \forall p, q \in X, x \in R .
$$

Corollary 4.3 (see [12]). Let $(X, d)$ be a complete metric space, and let $f: X \rightarrow(-\infty, \infty]$ be a proper lower semicontinuous function, bounded from below. Assume that for any $u \in X$ with $\inf _{v \in X} f(v)<f(u)$, there exists $w \in X$ with $w \neq u$ and $f(w)+d(u, w) \leq f(u)$. Then there exists $w_{o} \in X$ such that $\inf _{v \in X} f(v)=f\left(w_{0}\right)$.

Proof. Let $(X, d)$ be a complete metric space, and let $g: X \times X \rightarrow R^{+}, H: R \rightarrow R^{+}, F_{p, q}: R \rightarrow$ $[0.1](p, q \in X)$, and $t:[0,1] \times[0,1] \rightarrow[0,1]$ be mappings such that

$$
\begin{gathered}
g(p, q)=d(p, q) \quad \forall p, q \in X, \\
H(x)=0 \quad \text { if } x \leq 0, \quad H(x)=1 \quad \text { if } x>0, \\
F_{p, q}(x)=H(x-d(p, q)) \quad \forall p, q \in X, x>0, \\
t(a, b)=\min \{a, b\} \quad \forall a, b \in[0,1] .
\end{gathered}
$$

Then, all conditions of Corollary 4.3 satisfy all conditions of Lemma 4.1. Therefore, result of Corollary 4.3 follows from Lemma 4.1 .

The following example shows that Theorem 4.2 is more general than Corollary 4.3.

Example 4.4. Let $H, F$, and $L$ be the same as in Theorem 4.2. Let $X=[0,3]$ be the closed interval with the usual metric, $k=1 / 2$, and let $g: X \times X \rightarrow R^{+}, F: X \times X \rightarrow L$, and 
$f: X \rightarrow(-\infty, \infty]$ be mappings defined as follows:

$$
\begin{gathered}
g(u, w)=w \quad \forall u, w \in X, \\
{[F(u, w)](x) \equiv F_{u, w}(x)=H(x-|u-w|) \quad \forall u, w \in X, x>0,} \\
f(u)= \begin{cases}0, & 0 \leq u \leq 1 \\
-3 u+7, & 1<u<2 \\
2 u-2, & 2 \leq u \leq 3 .\end{cases}
\end{gathered}
$$

Then, for any $u \in X$ with $\inf _{v \in X} f(v)<f(u)$, there exists $w=1 \in X-\{u\}$ such that

$$
\begin{gathered}
g(u, w) \leq f(u)-f(w), \\
H(k x-|u-w|) \geq H\left(x-\frac{1}{k}[f(u)-f(w)]\right) \quad \forall x>0 .
\end{gathered}
$$

Let $\left\{u_{n}\right\}$ be a sequence of $X$ such that $\lim _{n \rightarrow \infty} u_{n}=0$. Then, clearly conditions (1), (2), (3), and (4) in Lemma 4.1 are satisfied. Thus there exists $0 \in X$ such that

$$
\inf _{v \in X} f(v)=f(0) .
$$

Therefore, all conditions of Theorem 4.2 are satisfied. Since $f$ is not lower semicontinuous at $u=2$, and $g$ is not metric, Corollary 4.3 cannot be applicable.

Theorem 4.5. Suppose that condition (4) in Lemma 4.1 is replaced with the following conditions. For self-mapping $T$ on $X$,

$$
\begin{gathered}
g(u, T u) \leq f(u)-f(T u) \quad \forall u \in X, \\
F_{u, T u}(k x) \geq H\left(x-\frac{1}{k}[f(u)-f(T u)]\right) \quad \forall u \in X, x>0 \text { and some } k \in(0,1) .
\end{gathered}
$$

Then, $T$ has a fixed point in $X$.

Proof. Suppose $u \neq T u$ for all $u \in X$. Then by Lemma 4.1, there exists $w_{0} \in X$ such that

$$
f\left(w_{0}\right)=\inf _{v \in X} f(v)
$$

Since $g\left(w_{0}, T w_{0}\right)+f\left(T w_{0}\right) \leq f\left(w_{0}\right)$, we have

$$
\begin{aligned}
f\left(T w_{0}\right) & =f\left(w_{0}\right)=\inf _{v \in X} f(v), \\
F_{w_{0}, T w_{0}}(k x) & \geq H\left(x-\frac{1}{k}\left[f\left(w_{0}\right)-f\left(T w_{0}\right)\right]\right) \\
& =H(x) \geq F_{w_{0}, T w_{0}}(x), \quad \forall x>0 \text { and some } k \in(0,1) .
\end{aligned}
$$


By the same method as in proof of Lemma 4.1, it follows that $w_{0}=T w_{0}$. But this contradicts our assumption that $u \neq T u$ for all $u \in X$. The proof of Theorem 4.5 is complete.

Theorem 4.6. Let $(X, d)$ be a metric space, $T: X \rightarrow X$, and let $H, g$, and $f$ be satisfied conditions (1), (2), and (3) in Lemma 4.1. Suppose that

$$
\begin{gathered}
g(u, T u) \leq f(u)-f(T u) \quad \forall u \in X, \\
H(k x-d(u, T u)) \geq H\left(x-\frac{1}{k}[f(u)-f(T u)]\right) \quad \forall x>0 \text { and some } k \in(0,1) .
\end{gathered}
$$

Then, $T$ has a fixed point in $X$.

Proof. By method similar to Theorem 4.2, the result of Theorem 4.6 follows.

Corollary 4.7 (see [7]). Let $(X, d)$ be a complete metric space, and let $f: X \rightarrow(-\infty, \infty]$ is a proper lower semicontinuous function bounded from below. Let $T$ be a mapping from $X$ into itself such that

$$
d(u, T u) \leq f(u)-f(T u) \quad \forall u \in X
$$

Then, $T$ has a fixed point in X.

Proof. By the same method as in Corollary 4.3, the result of Corollary 4.7 follows.

The following example shows that all conditions of Theorem 4.6 are satisfied but not that of Corollary 4.7 .

Example 4.8. Let $X, F, H, g$, and $k$ be the same as in Example 4.4. Suppose that $f: X \rightarrow$ $(-\infty, \infty]$ and $T: X \rightarrow X$ are mappings defined as follows:

$$
f(x)=\left\{\begin{array}{ll}
\frac{3}{2} x, & \text { if } 0 \leq x<2, \\
2 x, & \text { if } 2 \leq x \leq 3
\end{array} \quad \text { Tx }=\frac{1}{2} x \quad \forall x \in X\right.
$$

Then clearly, all conditions of Theorem 4.6 are satisfied but not that of Corollary 4.7 , since $f$ is not lower semicontinuous at $x=2$.

Natural question arises from Example 3.5.

Question 1. Whether Theorem 3.7 would remain true if (i), (ii), and (iii) in Theorem 3.7 are substituted by some suitable conditions?

\section{References}

[1] K. Menger, "Statistical metrics," Proceedings of the National Academy of Sciences of the United States of America, vol. 28, pp. 535-537, 1942.

[2] B. Schweizer and A. Sklar, Probabilistic Metric Spaces, North-Holland Series in Probability and Applied Mathematics, North-Holland Publishing, New York, NY, USA, 1983. 
[3] M. S. El Naschie, "On the uncertainty of Cantorian geometry and the two-slit experiment," Chaos, Solitons and Fractals, vol. 9, no. 3, pp. 517-529, 1998.

[4] M. S. El Naschie, "A review of E infinity theory and the mass spectrum of high energy particle physics," Chaos, Solitons and Fractals, vol. 19, no. 1, pp. 209-236, 2004.

[5] M. S. El Naschie, "The idealized quantum two-slit gedanken experiment revisited-Criticism and reinterpretation," Chaos, Solitons and Fractals, vol. 27, no. 4, pp. 9-13, 2006.

[6] L. B. Cirić, "A generalization of Banach's contraction principle," Proceedings of the American Mathematical Society, vol. 45, pp. 267-273, 1974.

[7] J. Caristi, "Fixed point theorems for mappings satisfying inwardness conditions," Transactions of the American Mathematical Society, vol. 215, pp. 241-251, 1976.

[8] L. B. Ćirić, "Contractive type non-self mappings on metric spaces of hyperbolic type," Journal of Mathematical Analysis and Applications, vol. 317, no. 1, pp. 28-42, 2006.

[9] L. B. Cirić, "Solving the Banach fixed point principle for nonlinear contractions in probabilistic metric spaces," Nonlinear Analysis, vol. 72, no. 3-4, pp. 2009-2018, 2010.

[10] Y. Feng and S. Liu, "Fixed point theorems for multi-valued contractive mappings and multi-valued Caristi type mappings," Journal of Mathematical Analysis and Applications, vol. 317, no. 1, pp. 103-112, 2006.

[11] M. A. Khamsi, "Remarks on Caristi's fixed point theorem," Nonlinear Analysis, vol. 71, no. 1-2, pp. 227-231, 2009.

[12] W. Takahashi, "Minimization theorems and fixed point theorems," in Nonlinear Analysis and Mathematical Economics, T. Maruyama, Ed., pp. 175-191, RIMS Kokyuroku 829, 1993.

[13] J. S. Ume, "Fixed point theorems related to Cirić contraction principle," Journal of Mathematical Analysis and Applications, vol. 225, no. 2, pp. 630-640, 1998.

[14] J. S. Ume, "Variational principles, minimization theorems, and fixed-point theorems on generalized metric spaces," Journal of Optimization Theory and Applications, vol. 118, no. 3, pp. 619-633, 2003.

[15] J. S. Ume, "Extensions of minimization theorems and fixed point theorems on a quasimetric space," Fixed Point Theory and Applications, Article ID 230101, pp. 1-15, 2008.

[16] J. S. Ume, "Existence theorems for generalized distance on complete metric spaces," Fixed Point Theory and Applications, Article ID 397150, pp. 1-21, 2010.

[17] S. N. Mishra, "Common fixed points of compatible mappings in PM-spaces," Mathematica Japonica, vol. 36 , no. 2 , pp. $283-289,1991$.

[18] O. Hadzić, "A fixed point theorem in Menger spaces," Publications of Institut Mathématique, vol. 26(40), pp. 107-112, 1979.

[19] O. Hadzić, "Fixed point theorems for multivalued mappings in probabilistic metric spaces," Fuzzy Sets and Systems, vol. 88, no. 2, pp. 219-226, 1997.

[20] V. M. Sehgal and A. T. Bharucha-Reid, "Fixed points of contraction mappings on probabilistic metric spaces," Mathematical Systems Theory, vol. 6, pp. 97-102, 1972. 


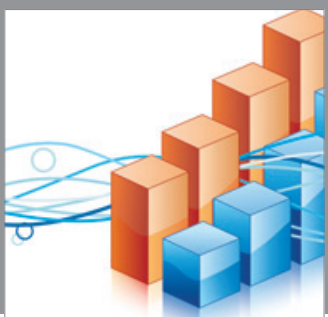

Advances in

Operations Research

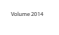

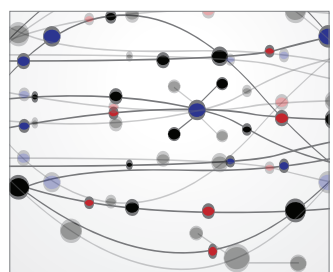

\section{The Scientific} World Journal
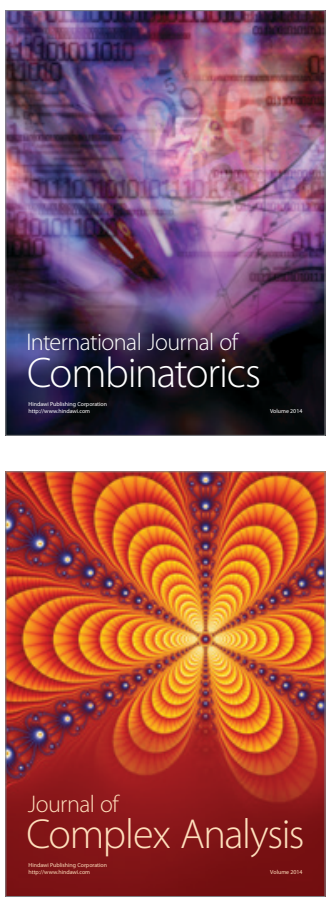

International Journal of

Mathematics and

Mathematical

Sciences
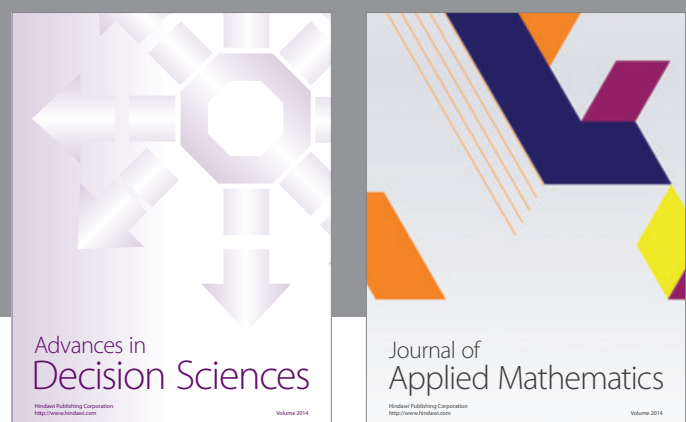

Journal of

Applied Mathematics
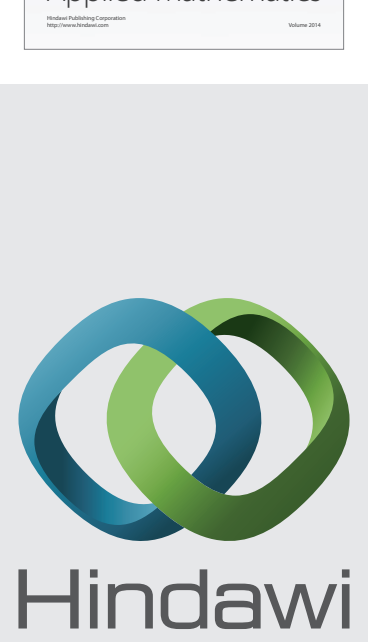

Submit your manuscripts at http://www.hindawi.com
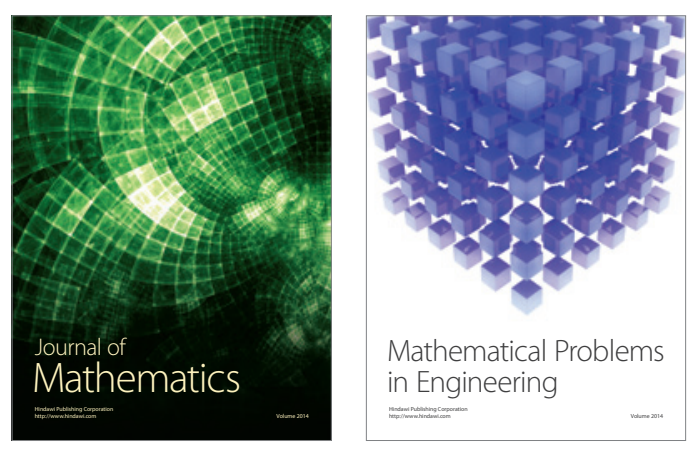

Mathematical Problems in Engineering
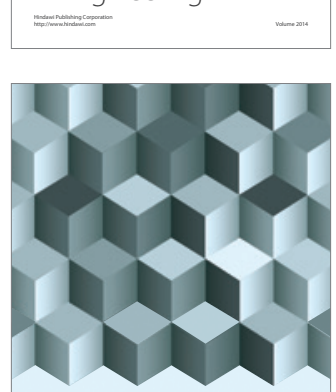

Journal of

Function Spaces
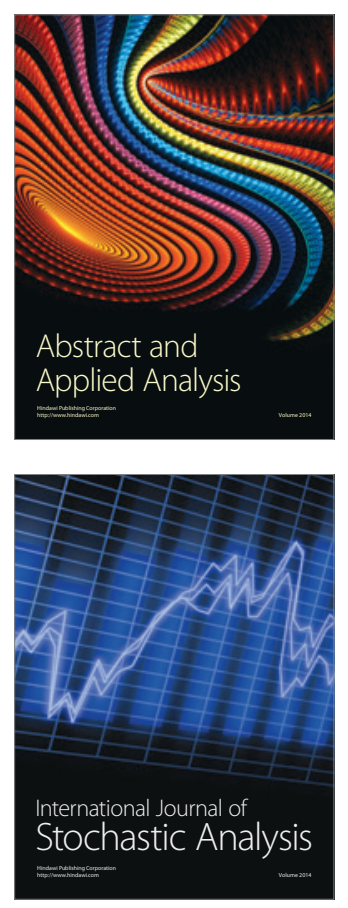

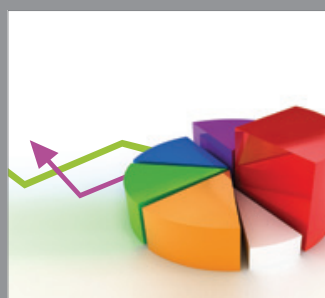

ournal of

Probability and Statistics

Promensencen
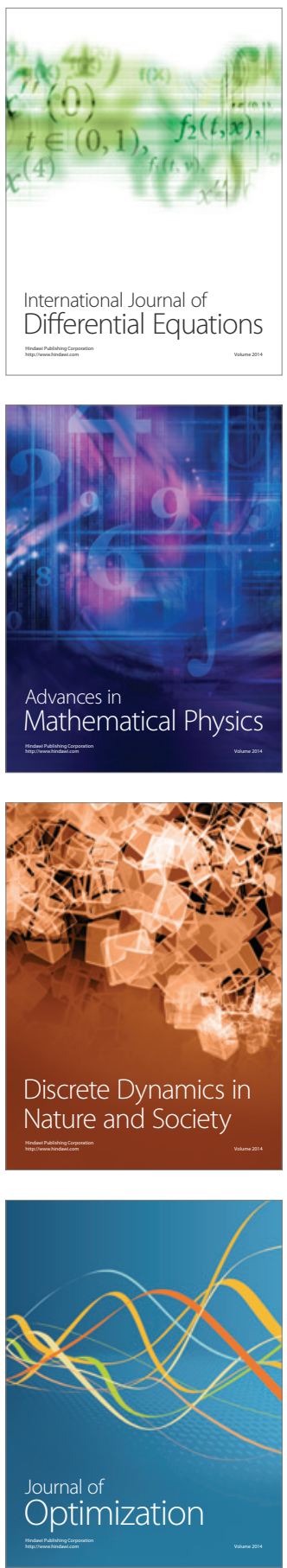